\title{
A Non-Iterative Method for Locating Soft Faults in Complex Wire Networks
}

\author{
Layane Abboud, Student Member, IEEE, Andrea Cozza, Senior Member, IEEE, and Lionel Pichon
}

\begin{abstract}
Reflectometry-based methods are the standard choice for fault detection techniques in wire networks. While effective when dealing with simple networks and relatively hard faults, their results can be of more difficult interpretation if a network presents more than two branches. In this paper we propose the use of an alternative technique based on a coherent multi-port characterization of a network under test. The data thus collected are used to define excitation signals that will be focusing over the position of a fault, following a method already successfully applied in geophysical prospection techniques and non-destructive testing, namely the DORT method, based on the synthesis of time-reversed signals. It is shown that a direct transposition of this technique to wire networks is not possible, due to the guided nature of wave propagation in wire networks, leading to the impossibility of assuming a dominant direction of propagation, as opposed to the case of propagation in open media. A differential version of the DORT method is introduced, enabling an accurate identification of the original position of faults. Numerical and experimental results are presented to demonstrate the feasibility of this approach.
\end{abstract}

Index Terms-Fault location, soft faults, complex wire networks, non-iterative methods, time-reversal operator.

\section{INTRODUCTION}

Due to their ubiquitous use in any medium/large sized infrastructure (e.g., buildings, transport systems, vehicles) and their primary role in energy and signals distribution, wire networks are fundamental subsystems whose correct functioning is of critical importance. Ensuring their reliable use requires the availability of techniques capable of detecting the presence of faults that could potentially put in jeopardy the infrastructures relying on these networks.

While a large number of such techniques has been devised in the past, the most widely applied are certainly reflectometrybased techniques [1], [2]. Reflectometry techniques allow relatively simple test setups for the analysis of a network under test (NUT); typically, a single test port is used in order to inject a test signal into the NUT. This signal will then propagate along a transmission line without coming back at the starting point unless encountering an impedance discontinuity, e.g., a branch junction, a load or a local unwanted modification of the line, i.e., a fault. When these modifications involve hard faults, e.g., a short or an open circuit, test signals are completely

L. Abboud and A. Cozza are with the Département de Recherche en Électromagnétisme, Laboratoire des Signaux et Systèmes (L2S), UMR 8506 SUPELEC - Univ Paris-Sud - CNRS, 3 rue Joliot-Curie, 91192 Gif-sur-Yvette, France.

L. Pichon is with the Laboratoire de Génie Électrique de Paris (LGEP), UMR 8507 SUPELEC - Univ Paris-Sud - CNRS, 11 rue Joliot-Curie, 91192 Gif-sur-Yvette, France.

Contact e-mail: andrea.cozza@supelec.fr reflected back towards the test port; the strong rate of reflection helps in distinguishing between natural reflections (branches and loads) and hard faults. A large number of post-processing procedures allow to take a decision about the presence of a fault, its nature and eventually its position within the NUT [3], [4], [5], [6], [7].

Although reflectometry-based techniques are very effective and accurate when dealing with hard faults, the detection and location of other types of faults is more critical [8]. Of particular importance is the very large class of soft faults, characterized by weak reflectivities. The difficulties in their detection can be traced back to two basic phenomena: 1) shortterm modifications of the NUT topology, e.g., the distance between the conductors in a cable, can generate spurious reflections not related to any fault, but with an intensity that may be similar to that of certain soft faults [9], [10], [11]; this kind of situation occurs, e.g., when dealing with NUTs subject to mechanical vibrations; 2) spurious signals propagating throughout the NUT; these signals are not necessarily unwanted, as they can have a useful origin: e.g., communication signals transmitted through the NUT, used as a signal bus. This configuration occurs when considering live tests of the NUT. In both cases, weak echoes generated by soft faults can pass undetected with respect to these spurious signals propagating over the NUT.

In this paper we will focus onto the second scenario. Live testing is of practical importance, since it does not require turning off all the electronic equipments that normally use the NUT as a power or communication infrastructure; also known as embedded testing, this approach works in the background of the normal operation of the NUT. The structure of the NUT will also be assumed to be time-invariant.

Soft faults correspond to minor damages to the NUT, e.g., insulation alterations in the cables or partial cuts. Despite the fact that they are typically not as critical as hard ones, their potential degeneration into hard faults is a reason for investing in detection and location techniques capable of identifying them as soon as possible. Reflectometry-based techniques have been shown to be in difficulty in ensuring a reliable detection of soft faults [1], [12]; this state of affairs is worsened in the case of complex NUTs. A recent proposal in this direction has shown that using test signals matched, thanks to a timereversal approach, to the response of the NUT can significantly improve the probability of a proper detection in presence of noisy signals [13], especially when dealing with complex networks.

Reflectometry-based methods are also affected by another difficulty, namely the ambiguity in the interpretation of their 
results as soon as the NUT is composed of multiple branches connected through junctions, e.g., in tree-shaped structures. In practice, complex networks are more often found in practice than simple linear ones, e.g., in energy and signal distribution networks; still, most of the time fault-detection and location techniques are tested against simple NUTs [14], [15]. The direct use of reflectometry techniques in NUTs including junctions, e.g., Y-shaped networks, can properly detect the distance between the fault and the testing port, but cannot distinguish on what side the fault is located. Improved reflectometry techniques better suited to complex networks require the use of multiple testing ports, in order to solve the ambiguity [16].

Alternative approaches, still related to reflectometry techniques, have been recently developed; they are based on the iterative solution of an inverse problem, where the unknown is the position and nature of the fault [17]. In this case a model of the network is inserted within an iterative loop and the measured data are compared to those predicted by solving the direct model. An error minimization algorithm is then used to minimize the difference between the two data sets. The process is iterative and continues until a certain criterion is satisfied, such as a predefined error threshold. Such an approach can be very time consuming and depends on the complexity of the network.

From a formal point of view, the problem of soft-fault location in a complex wire network is closely related to the problem of target location encountered in radar detection: increasing multiple scattering in the medium poses a significant difficulty when the target's signature is weak compared to other scatterers. To deal with this difficulty time-reversal techniques can ensure a maximization of the fault-related echo, by maximizing the energy impinging on the fault position with respect to the rest of the system under test. This property has been exploited in medical imaging [18], radar and sonar communications [19], [20] and the sensing of buried objects [21], [22]. For most of these imaging applications, analysis of the time-reversal operator, or DORT, [23] is fundamental.

This paper aims to show that this kind of techniques can be adapted to the problem of soft-fault location in wire networks. The proposed method involves two major differences with respect to existing TDR-based techniques: 1) multiple testing ports are used, but in a new perspective: unlike distributed reflectometry [16] where the results obtained from each testing ports are independently processed and subsequently crosschecked to solve ambiguities, the proposed method allows the simultaneous analysis of all multi-port data to the location of the fault; 2) the signals applied to the NUT no longer aim at directly locating the fault, but are rather meant to characterize the propagation of signals through the NUT. The eventual presence of a fault is subsequently inferred from the scattering matrix of the NUT, rather than from echoes, as in TDR techniques. The proposed approach is therefore radically different from TDR-based techniques.

The increased rate of information thus extracted from the NUT leads to a more effective solution for soft-fault location in complex networks. The intrinsical non-iterative definition of DORT-based techniques also implies a direct solution that could be better suited to real-time monitoring of networks.
The paper starts by recalling the main steps behind the DORT method in section II, in particular how to define excitation signals for selective focusing in complex media, while discussing the main reasons why this kind of technique, though very effective in open media, cannot be directly transposed to NUTs, where waves are guided rather than radiated. The modifications introduced in section III are shown to lead to the ability to focus energy over a fault, paving the way to an alternative fault-location technique. Numerical and experimental examples are presented in section IV, illustrating how the proposed technique leads to a simpler decision process.

\section{Selective FocUsing IN COMPLEX MEDiA}

An entirely different approach to fault location can be proposed by observing that a fault in wire networks is nothing else than a discontinuity, i.e., a scatterer, typically of dimensions well below a wavelength. This simple observation implies that identifying the position (and eventually the nature) of a fault is fundamentally the same problem than locating a scatterer in a generic medium. A large array of techniques have been proposed over the years to locate scatterers; e.g., we can mention the extended family of inverse techniques [24], based on the use of direct propagation models coupled to optimization techniques. All these techniques share a common feature, the use of multi-port scattering data as the starting point.

The parallel between the problem of fault location and inverse problems also implies that the use of reflectometry techniques is not necessarily the best choice, as their seldom use in inverse problems attests. Inverse techniques based on multi-port scattering data are inevitably more efficient and accurate, since based on the simultaneous use of all available data, rather than a collection of separate reflectometry tests (see, e.g., [16]).

Within the context of this paper, we are rather interested in an alternative and relatively recent technique, based on timereversal focusing of waves. This technique is usually referred to as DORT, a French acronym standing for expansion of the time-reversal operator; it was initially introduced for the generation of wavefronts automatically focusing over scatterers smaller than a wavelength [23], while its potential for scatterer location was proposed and developed in subsequent work [25], [26].

The tenets of the DORT technique are briefly recalled in II-A, while in II-B we argue about the obstacles that stand in the way of its direct transposition to the case of fault detection in wire networks, detailing the reasons for our proposing a heuristic location criterium detailed in section III. This kind of techniques are often presented in the frequency domain, but this choice should not be regarded as a limitation. The proposed method requires the use of non-harmonic signals in order to define an origin in space and time; this notwithstanding, rather than directly working in the time domain, it will be by far simpler to start our analysis in the frequency domain, while switching back to the time domain afterwards. 


\section{A. The DORT technique for waves radiated in open media}

The DORT requires the presence of multiple transducers around the scatterer: incident signals $a_{i}(\omega)$ are applied to the port of the $i$-th transducer, or testing port, generating test wavefronts that will eventually be scattered by a scatterer in front of the transducers and recorded as output signals $b_{j}(\omega)$ over each testing port. These data can be reshaped into a scattering matrix, following the standard definition used in microwave theory [27]. Arranging the incoming and outgoing signals observed at each port into two vectors, $\boldsymbol{a}(\omega)$ and $\boldsymbol{b}(\omega)$, respectively, the scattering matrix $\boldsymbol{S}(\omega)$ is given by

$$
\boldsymbol{b}(\omega)=\boldsymbol{S}(\omega) \boldsymbol{a}(\omega) .
$$

By introducing the time-reversal operator matrix $\boldsymbol{K}(\omega)$

$$
\boldsymbol{K}(\omega)=\boldsymbol{S}(\omega) \boldsymbol{S}^{\dagger}(\omega),
$$

where $\dagger$ stands for the Hermitian transpose, it can be shown (see [23]) that the eigenvectors corresponding to the most significative eigenvalues of $\boldsymbol{K}(\omega)$ provide excitation signals that once applied to the transducers array will generate wavefronts focusing on a scatterer in front of the array.

The ability to generate wavefronts focusing over a scatterer can be straightforwardly converted into a scatterer-locating technique. To this end, rather than applying the excitation signals to the transducer array, their propagation through a given medium can be simulated numerically, by using theoretical models of wave propagation in the medium unperturbed by the scatterer. By monitoring the propagation of the focusing wavefront, the position of their maximal intensity, i.e., their focal region, indicates the position of the scatterer. This kind of approach, eventually coupled with sub-space techniques such as MUSIC, has been adopted in geological prospection techniques, with excellent results in terms of location accuracy and reliability [26], [28].

The use of a propagation model without taking into account the presence of the scatterer requires that the latter behave as a weak perturbation, i.e., that the amount of energy it scatters be negligible with respect to the incident energy. This condition, transposed to the case of fault-location in wire networks, perfectly fits the characteristics of soft faults. Thanks to the close similarity between fault location in complex wire networks and scatterer location in complex media, it is reasonable to expect that a DORT-based location method could also provide an improved performance with respect to standard reflectometry techniques.

\section{B. The case of wire networks}

The use of focusing signals is schematically depicted in Fig. 1, where the multi-port response of an NUT is measured through three testing ports. Signals defined by means of the DORT method (see section III) could be applied to these same ports, leading to the expected focusing of guided waves from the two sides of the branch containing the fault.

This simple example already points to one of the reasons why the DORT technique cannot be directly applied to wire networks. In fact, while the two waves converge onto the fault

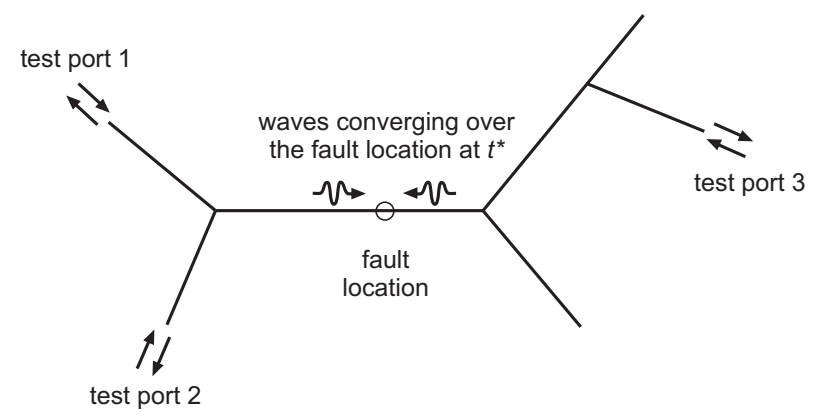

Fig. 1: A schematic illustration of how focusing signals can be used to locate a fault in a wire network. DORT-based signals are applied to three excitation ports in this example, and lead to guided waves converging over the fault location at the time $t^{*}$. For $t>t^{*}$ the same signals will continue propagating over the opposite sides of the fault and after multiple reflections over the NUT discontinuities (e.g., junctions), will eventually cross again to form unwanted focal spots.

location at the time $t^{*}$, for $t>t^{*}$ they will continue propagating towards opposite directions, where they will inevitably interact with discontinuities such as junctions. As a result, they will be reflected back and transmitted over other parts of the NUT, where they could eventually interfere constructively again, yielding artefact focal regions. Since the most intuitive way of deciding if a focusing is under way is to check if two waves propagating in opposite directions cross their common paths interfering constructively, if for $t>t^{*}$ stray signals could do the same it would be hardly possible to distinguish whether a crossing has occurred at the location of a fault, or just by chance of multiple-scattering interactions after the first focusing at the fault location.

The potential and unpredictable appearance of stray focal regions is worsened by one more difference existing between radiated and guided propagation: the intensity of radiated waves diminishes because of divergent propagation, with energy spreading over ever larger regions of space, while guided waves can only change in intensity if the guiding structure is lossy. Hence, the chances of stray signals interfering as if focusing while maintaining an intensity similar to the true focal region is much higher in wire networks than in an open medium.

Another major difference is that while in open media all the signals received at the transducers are originated by scattered fields, with a negligible contribution from the original test waves generated by the test ports, in a wire network output signals often involve a mix of the two. This situation is illustrated in Fig. 2, where the signal received over the $j$-th port results from the transmission of the test signal applied at port $i$-th through the fault. Since the reflected and transmitted signals are intrinsically different, the fault response will appear to differ depending on the test port under consideration. This situation has no analogy in open media, making the use of the DORT method in guiding media critical. 


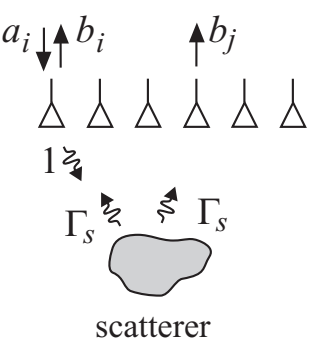

(a)

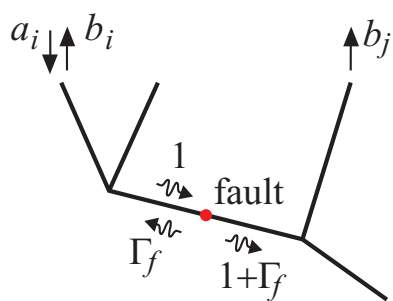

(b)
Fig. 2: Comparison of the propagation phenomena underlying the multi-port responses of : (a) an electrically small scatterer in an open medium, with reflectivity $\Gamma_{s}$; (b) a fault in a wire network with reflectivity $\Gamma_{f}$. In both cases the $j$-th output signal is generated by exciting the $i$-th testing port with a unitary test signal; while in (a) the signal $b_{j}$ is basically related to the scattered field, in (b) it is rather related to the total transmitted field, while the output signal observed at the $i$-th port will be related to the reflected signal.

\section{LOCATING SOFT FAULTS IN COMPLEX NETWORKS}

In order to access the benefits of the DORT method it is first necessary to make sure that all output signals from the multiport characterization be only related to signals scattered by the fault rather than transmitted through it from one testing port to another. As schematically shown in Fig.3, this can be done by first introducing a preliminary characterization of a faultless version of the NUT, taken as a reference against which subsequent tests are compared. This reference data is usually referred to as a baseline measurement. The rationale for this method is based on the following observation: while echoes from soft faults are often dwarfed by those generated by, e.g., junctions, a baseline measurement contains exactly the same echoes from the same junctions; taking the difference of the two results is intended to cancel out the contribution from the NUT itself (i.e., the junctions in this example), making the fault response detectable. Baselining is indeed widely used in reflectometry-based methods as a contrast enhancing technique, rather than for the sake of accessing the scattering response of an eventual fault, as here required.

Although this kind of approach intrinsically requires a timeinvariant NUT, it allows a more clear identification of signals scattered by the fault. From a propagation point of view, the use of baseline-based methods imply a Born approximation [29], where the total energy of waves scattered by the fault is assumed to be negligible with respect to the energy of waves impinging on the scatterer; in other words, the fault is required to behave as a weak perturbation in the propagation of waves within the NUT. While this assumption does not hold in the case of hard faults, it is accurate when dealing with soft faults, thanks to their weak reflectivity, as recalled in the introduction. The rationale for accepting this approach is that while location techniques for hard faults are widely available, reliable location of soft faults is a harder task, particularly in complex NUTs in a noisy electromagnetic environment.

According to this paradigm, the scattering matrix appearing

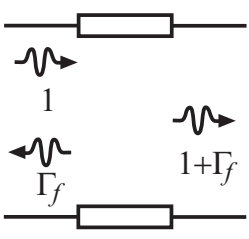

(a)

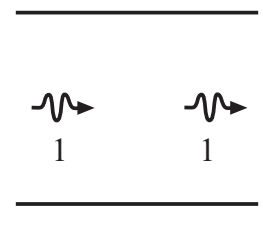

(b)

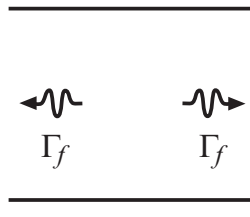

(c)
Fig. 3: Wave scattering from a fault in a transmission line, represented as a lumped discontinuity: (a) reflection and transmission coefficients in presence of a fault and (b) for the same line without fault; (c) equivalent representation of the fault as a secondary source, after subtracting (b) from (a). The last case implies the use of an unperturbed line as valid, as long as $\left|\Gamma_{f}\right| \ll 1$.

in (2) cannot be measured directly in a single step, but rather needs to be computed from a two-step procedure. The first step involves measuring the baseline scattering matrix $\boldsymbol{S}_{b}(\omega)$, for a faultless version of the NUT; this operation can be done, e.g., before putting the NUT in operation. The second step is the actual test on an eventually faulty NUT, yielding the corresponding matrix $\boldsymbol{S}(\omega)$. The resulting scattering matrix $S_{s}(\omega)=S(\omega)-S_{b}(\omega)$ now only contains data related to signals scattered by a fault. The result of this operation is equivalent to observing the signals scattered by the fault as though it were an equivalent, or secondary, source (Fig. 3(c)). It is therefore natural to expect that $S_{s}(\omega)$ yield direct information about the position of a fault, as demonstrated experimentally in the next section.

The equivalent scattering matrix $S_{s}(\omega)$ obtained from the excitation of the NUT can be used in order to synthesize test signals intended to focus over the fault position. Assuming the presence of a single fault, the associated time-reversal operator $\boldsymbol{K}_{s}(\omega)$ will present a single significative eigenvalue over the entire frequency of interest; the corresponding eigenvector will be referred to as $\boldsymbol{w}=\left[w_{1}(\omega), \ldots, w_{N}(\omega)\right]^{\mathrm{T}}$, where $N$ is equal to the number of testing ports.

Since the proposed location method is based on the monitoring of focusing signals, an accurate location of the fault requires the use of signals with the shortest possible spatial support. This issue is at the basis of all reflectometry methods, and fundamentally requires to chose focusing signals with a time-dependence $p(t)$ according to the precision required by the test user. The use of wide-band pulsed focusing signals allow an easier identification of the focal region; this is especially true in complex NUTs, where multiple reflections over the junctions can make the identification of the focal region far from trivial.

In our proposal, the testing signals applied to each of the test ports of the NUT models are defined as

$$
x_{i}(t)=\int P(\omega) w_{i}(\omega) \mathrm{e}^{\mathrm{j} \omega t} \mathrm{~d} \omega,
$$

where the integral is taken over the bandwidth of the Fourier spectrum $P(\omega)$ of $p(t)$.

An example of the results obtained by implementing these ideas is shown in Fig. 4. The Y-shaped NUT in Fig. 4(a) 
(a)

(1)
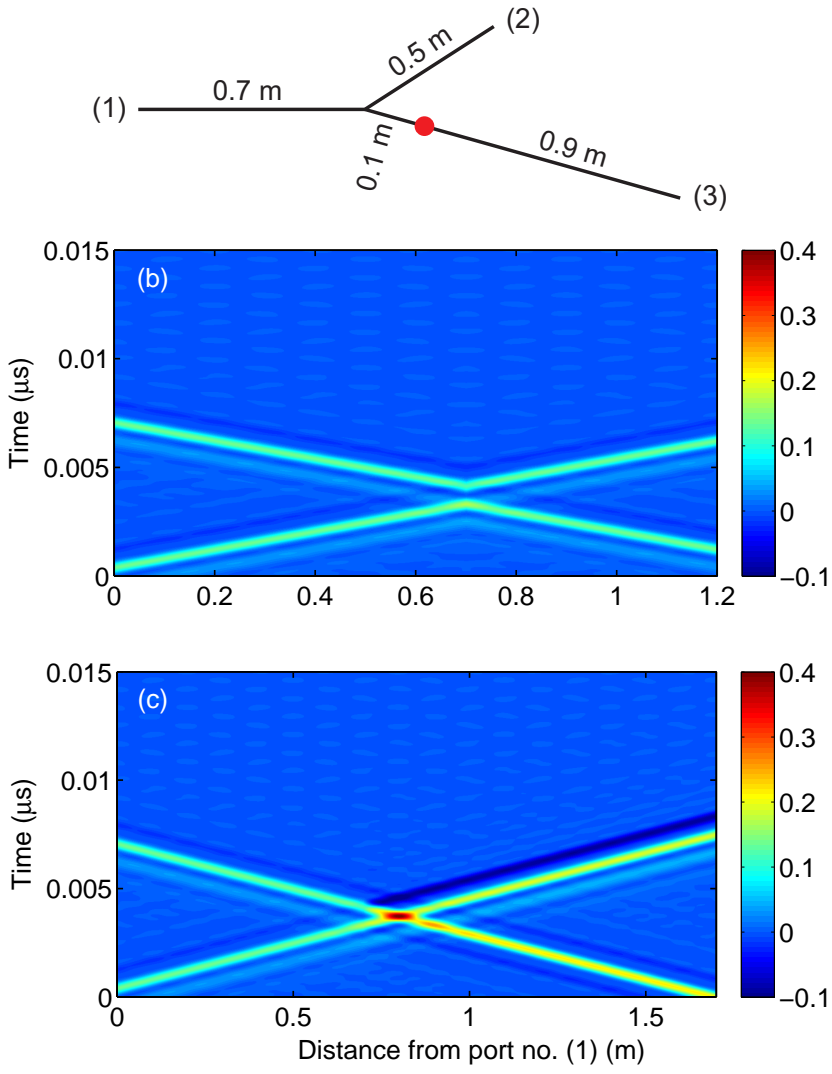

Fig. 4: An example of differential DORT fault location: (a) the NUT, with the fault position indicated by the red dot and all ports impedance-matched; space-time propagation diagrams of the signal (voltage waves) excited by the differential DORT method, as recorded (b) along the path (1)-(2) and (c) along the path (1)-(3). The fault is easily located by looking for the only focal region generated by the signals, where the maximal intensity is observed.

was considered, with a fault located $0.1 \mathrm{~m}$ away from the junction and modeled, for the sake of simplicity, by a parallel resistance of $400 \Omega$, i.e., with a reflection coefficient equal to -0.058 for transmission lines with a characteristic impedance equal to $50 \Omega$. The choice of a resistive model for the fault can be shown to have a minor effect on the performance of the proposed method; the case of a dispersive fault is discussed in section IV-B.

The scattering matrices for this example with and without the fault (baseline) were computed from transmission-line theory [30], assuming ideal non-dispersive lines, with a characteristic impedance equal to $50 \Omega$ and dimensions as shown in Fig. 4(a); we used an in-house code, but any SPICE-like model could be used to this effect. Excitation signals based on a truncated Gaussian pulse $p(t)$ (bandwidth equal to $800 \mathrm{MHz}$ ) derived as in (3) were applied to the three testing ports of a numerical model of the faultless NUT, monitoring their spacetime propagation within it. Figs. 4(b)-(c) present the space and time propagation of the test signals defined in (3), through the faultless NUT; the amplitude of the signals (voltage waves) are
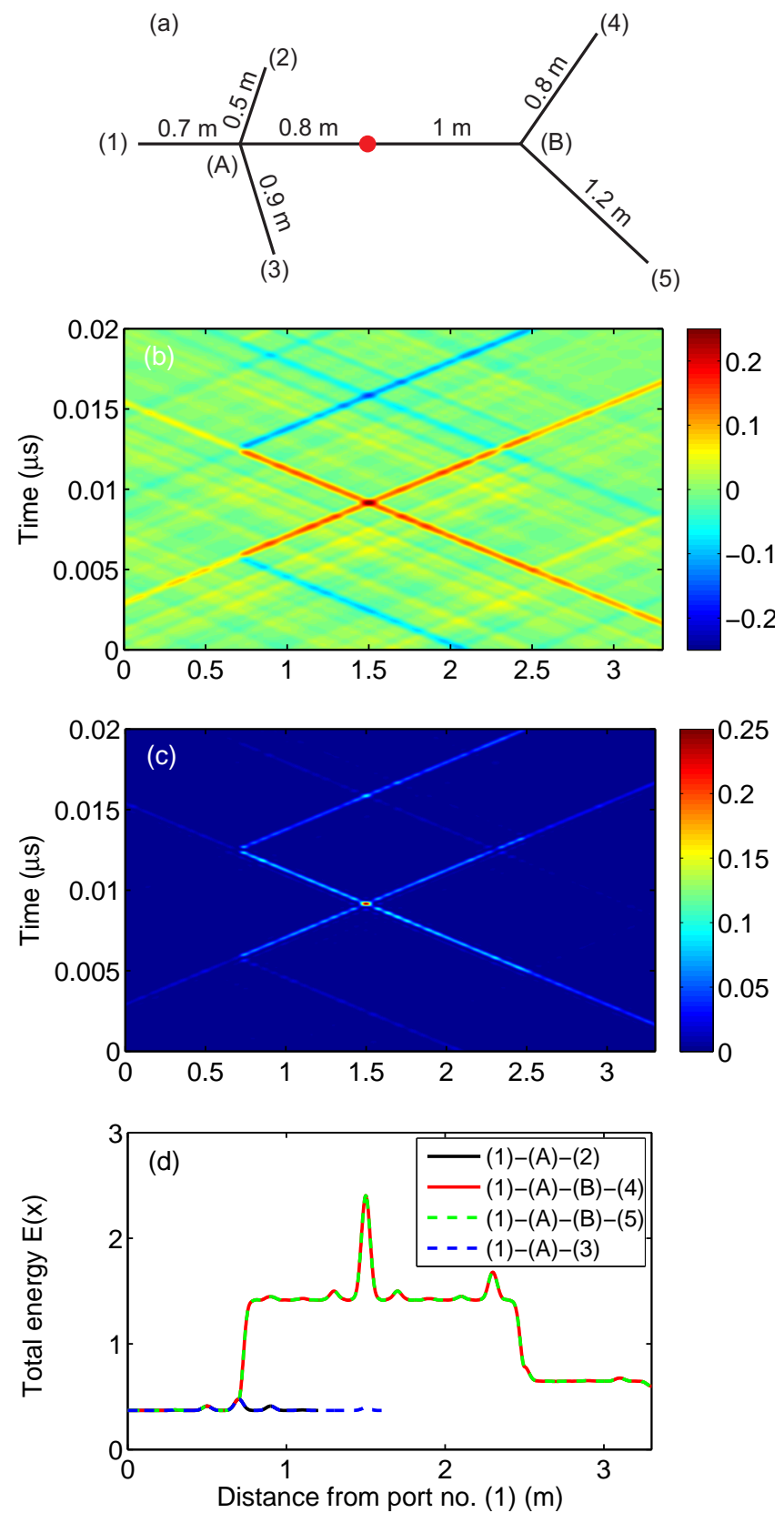

Fig. 5: Waves focusing onto the fault within a complex NUT: (a) the NUT; (b) voltage waves propagating through the path (1)-(A)-(B)-(4); (c) instantaneous power along the same path; (d) total energy observed along four different paths. Focusing waves are mainly observed along the branch where the fault is located and more easily identified in (c) than in (b).

indicated by the color scale. While on the one hand the signals applied to the three test ports do not lead to any constructive interference along the path (1)-(2) (Fig. 4(b)), on the other hand they strongly interfere along the path (1)-(3), giving place to an easily identifiable focal spot occurring at the position of the fault. In this simple example a visual inspection of the propagation is enough to unambiguously pinpoint the location of the fault; no focal region is generated in the branches where there is no fault. 
Unfortunately, as soon as more complex NUTs are considered, as those discussed in section IV, the interpretation of the results is by far less straightforward. Indeed, the likely appearance of focal regions generated by constructive interference of stray signals is the major issue: as opposed to the case of TDR techniques, time-reversed signals reflected by the fault do not correspond the first echoes, as shown in [13]. Actually, this is not an issue, since the proposed method does not locate faults by looking for the first significative echo, but rather seeks out the position within the NUT where the strongest focusing of energy occurs.

In order to distinguish the actual focal spot from spurious crossings due to constructive interferences of stray signals, it is necessary to take a closer look at the physical mechanisms involved. Focusing in a wave-guiding structure basically consists of two non-harmonic signals, typically pulses, propagating towards the fault position along opposite directions, converging over it at the same time $t^{*}$. These two signals have not necessarily the same amplitude, since in general they are associated with different test ports and travel along parts of a NUT with different topologies. Noting the voltages associated with these two signals as $v^{-}(x, t)$ and $v^{+}(x, t)$, respectively for the rightward and leftward propagating, the instantaneous power observed around the fault location is given by

$$
p(x, t)=Z_{c}^{-1}\left[v^{-}(x, t)+v^{+}(x, t)\right]^{2},
$$

where $Z_{c}$ is the characteristic impedance of the line where the fault occurs. The instantaneous power is an effective indicator of the occurrence of energy focusing, since in the focal region it will be higher than the sum of the individual instantaneous power contributions of the two terms in (4). In the case of equal-amplitude signals, the instantaneous power will be four times higher than that of each signal. As a reference, if the total amplitude were considered, only a twofold increase would be observed. The use of (4) therefore ensures a higher dynamics for the detection of the focal region.

The detection of the focal region, and therefore of the fault position, can also be greatly simplified by computing the total energy $E(x)$ observed at each position, as

$$
E(x)=\int p(x, t) \mathrm{d} t .
$$

The main advantage of this operation consists in the removal of the temporal dimension. An example of the result of this sequence of steps is shown in Fig. 5(b)-(d), for the NUT shown in Fig. 5(a), again affected by a $400 \Omega$ parallel fault; transmission lines and the pulse $p(t)$ share the same characteristics as in the previous example. While the use of (4) increases the visibility of the focal region, the time-integration further simplifies its identification. Fig 5(d) shows $E(x)$ for several propagation paths along the NUT; the strongest focusing occurs at the position of the fault, as expected from the discussions in the previous section.

For the sake of clarity, the steps required in the proposed method are summarized in the following list:

1) define a number $N$ of testing ports from which the NUT can be tested;
2) measure the scattering matrix $S_{b}(\omega)$ observed from the test ports before the NUT is in operation (baseline measurement);

3) the appearance of faults in the NUT can be tested by measuring the scattering matrix $S(\omega)$ observed from the same test ports;

4) compute the differential scattering matrix $S_{s}(\omega)=$ $\boldsymbol{S}(\omega)-\boldsymbol{S}_{b}(\omega)$ and the TR operator $\boldsymbol{K}_{s}(\omega)=$ $\boldsymbol{S}_{s}(\omega) \boldsymbol{S}_{s}^{\dagger}(\omega)$

5) solve $\boldsymbol{K}_{s}(\omega) \boldsymbol{v}_{k}(\omega)=\lambda_{k}(\omega) \boldsymbol{v}_{k}(\omega), \forall k \in[1, N]$ to find the eigenvalues and eigenvectors of $\boldsymbol{K}_{s}(\omega)$;

6) select the eigenvector $\boldsymbol{w}(\omega)$ corresponding to the largest eigenvalue;

7) compute from (3) the test signals $x_{i}(t)$;

8) monitor the time-domain propagation of the test signals in a numerical model of the faultless NUT;

9) compute the total energy $E(x)$ as in (5);

10) the position of the fault is given by argmax $E(x)$.

Steps 1) to 2) are only done once, in order to create a baseline data set used as a reference to check for the appearance of faults; the only measurement that is repeated in practice at each test is 3). The remaining steps occur in a post-processing setting.

\section{REsults}

The proposed method was applied to complex NUTs in numerical (IV-A) and experimental settings (IV-B). All the following results are based on the procedure summarized at the end of section III, characterizing the scattering matrix of the NUTs from DC up to $800 \mathrm{MHz}$. The pulse $p(t)$ appearing in (3) was chosen to have a truncated Gaussian Fourier-spectrum, with the truncation occurring at the $-3 \mathrm{~dB}$ bandwidth, equal to $800 \mathrm{MHz}$.

The following results are meant to illustrate the potential benefits provided by the differential DORT technique, adapted to complex wire networks. The sheer complexity of signal propagation through this kind of NUTs is a formidable obstacle to more general theoretical proofs. Since the basic idea behind any DORT method is to exploit the scattering nature of a fault and the time-reversal symmetry of wave propagation, there should be no doubt about the fact that DORT-based signals are bound to focus over the scatterer position, even in complex media. The large number of previous studies available in the literature should serve as a gauge of the validity of this working assumption (see, e.g., [26]).

In our investigations we made use of coaxial cables; the rationale was ensuring the reproducibility of the experimental results. This goal was enabled by the manufacturing of reference samples, affected by soft faults, as described in section IV-B. Clearly, the validity of the proposed method is not limited to coaxial cables; in fact, DORT approaches do just require the idea of finite-speed wave propagation in order to generate energy focusing in any medium. The same ideas apply to any kind of transmission line, even multiconductor ones. 


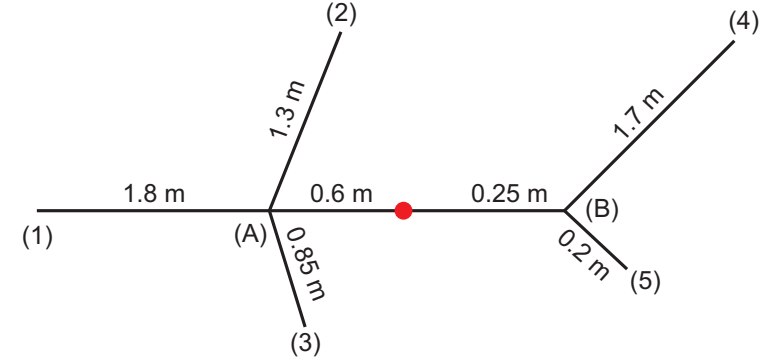

Fig. 6: The NUT considered for the analysis of the impact of a changing number of testing ports. Branches are not in scale.
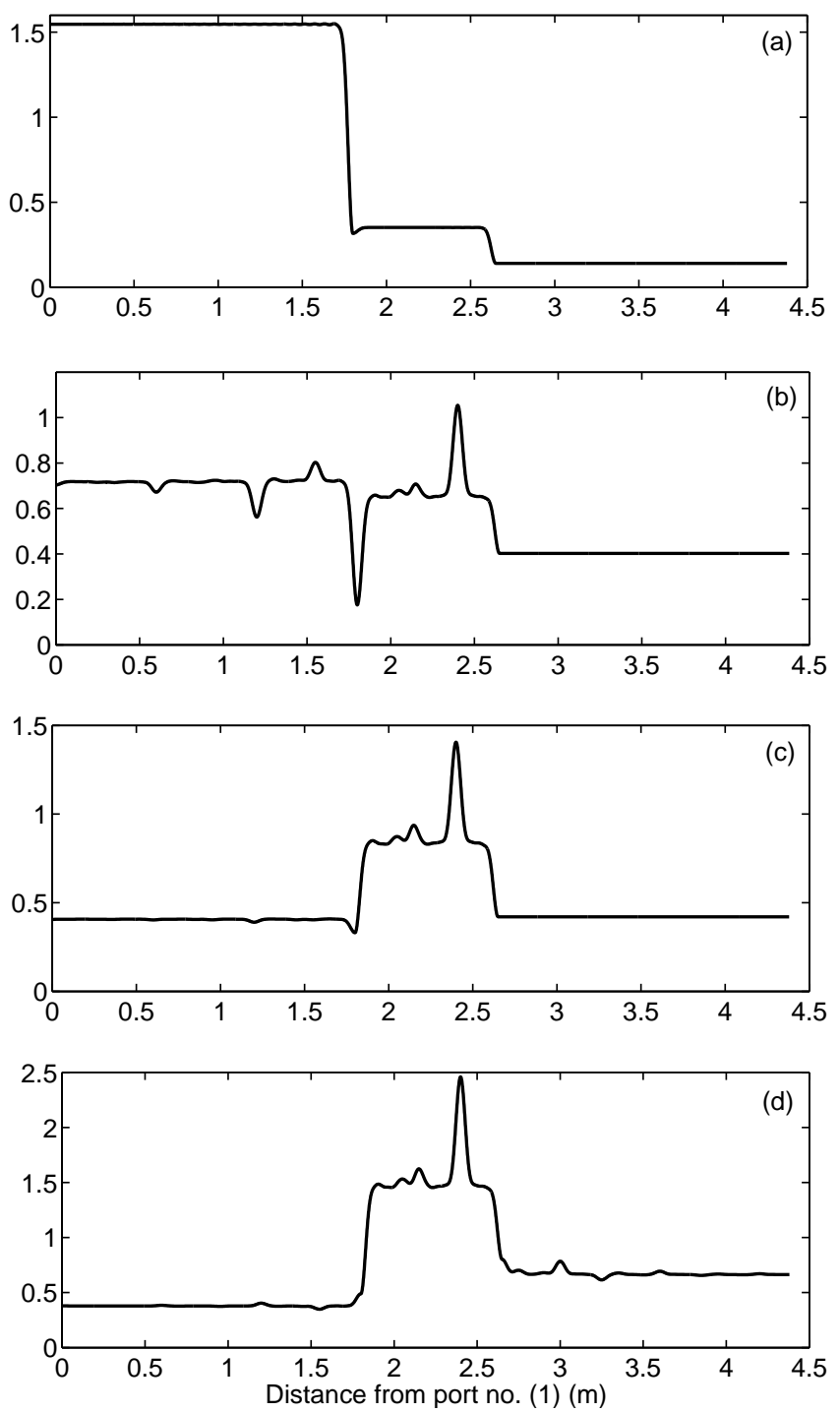

Fig. 7: The total energy $E(x)$ as estimated along the path from port (1) to (4) of the NUT in Fig. 6: (a) single-port excitation applied to (1); (b) two-port excitation at (1) and (5); (c) three-port excitation at (1), (2) and (5); (d) all NUT ends are connected to testing ports.

\section{A. Numerical results}

In this section we will consider the issue of how changing the number of testing ports affects the performance of the proposed method. To this end, the NUT shown in Fig. 6 was studied, once again by means of transmission-line theory; as already noted, any SPICE-like solver can be used to this effect.

The fault considered was a $400 \Omega$ parallel resistance as in the previous examples, and the characteristic impedance of the transmission lines making up the NUT was again equal to $50 \Omega$. The proposed procedure was applied to four different sets of testing ports detailed in Fig. 7, connected to the ends of the NUT. The scattering matrices of the NUT were computed twice, first by considering a faultless version (baseline) and then including the $400 \Omega$ fault. As explained in section III, the difference of these scattering matrices can be used in order to define testing signals focusing over the fault position. Depending on what testing ports are available for the test, different sets of excitation signals were computed by means of (3).

The total energy $E(x)$ estimated along the path linking port (1) and (4) is shown in Fig. 7. The first thing to notice is that a single-port excitation applied through port (1) does not lead to any focusing. This fact should not be surprising: in order to have focusing signals, they should propagate towards the fault along opposite directions. But for a single port this is not feasible, as most of the energy propagates from port (1) to the fault; no confusion should subsist on the eventual role of reflections of the excitation signal over junction (B) on the right: while indeed signals can be reflected back towards the left, they will appear at the fault position at a different time than those initially injected from (1). Hence, no focusing will be observed.

As soon as at least a second testing port is activated, signals defined by (3) will synchronize at the fault position. Fig. 7(b) presents the case where port (5), on the other side across the fault position, is used. In this case a focal region is easily spotted, coinciding with the fault position. Adding more testing ports improves the contrast between the total energy observed at the fault position and the background energy elsewhere; in fact, the improvement is more esthetic than instrumental to locating the fault, as the local contrast over the branch where the peak of $E(x)$ is found is hardly affected.

A simple conclusion, predictable with hindsight, is that in order to have focusing, and therefore locate the fault position, it is necessary to have at least two testing ports connected to the NUT in such a way as to have signals propagating towards the fault along opposite directions. This observation implies a clear limitation: since the position of the fault is initially unknown, how to chose where the testing ports should be connected? If faults need to be monitored on any possible position inside the NUT, than testing ports should be connected in such a way as to present direct (i.e., not relying on reflections) propagating paths covering all positions.

The position of the fault with respect to the NUT topology and the position of the testing ports has therefore a predictable behavior with clear consequences justified on physical 


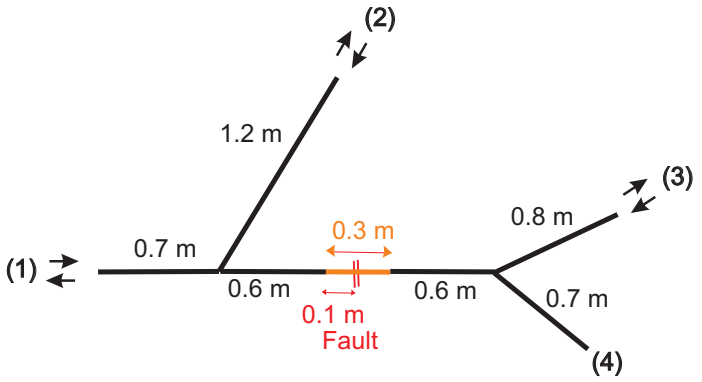

Fig. 8: Layouts of the NUT considered for the experimental validation. Each branch of the NUTs consisted of a flexible coaxial cable with a $50 \Omega$ characteristic impedance, while the red portions represent the samples containing the fault, as shown in Fig. 10. Three testing ports were considered; the line end (4) was either impedance-matched or opencircuited, depending on the studied case. The junctions were implemented by means of BNC T-junctions. The testing ports (double-arrow symbols) were connected to a vector network analyzer.

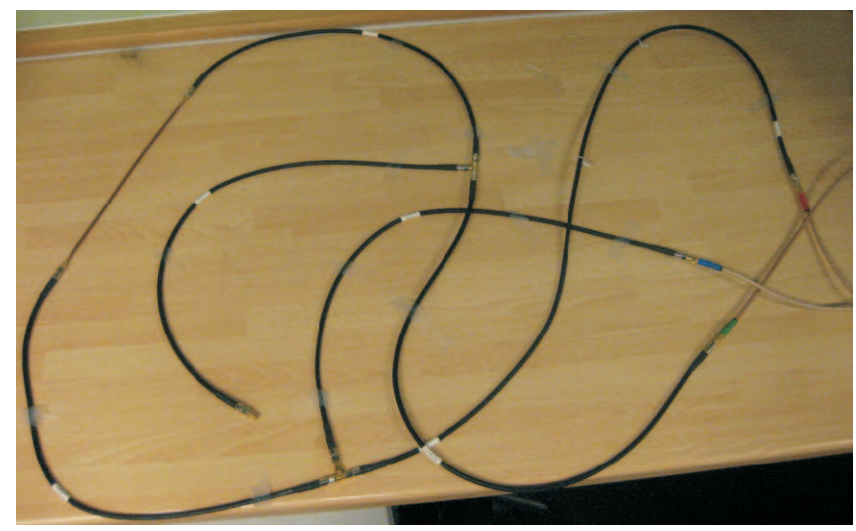

Fig. 9: Implementation of the NUT described in Fig. 8, as connected to a vector network analyzer for experimental tests. The $30-\mathrm{cm}$ long semi-rigid cable implementing the fault sample is visible in the upper-left corner while the three testing ports are found on the right.

grounds. As opposed to these observations, the relative position of the fault within the same branch can be expected to be negligible; as a matter of fact, from the point of view of the focusing signals, any shift of the fault along a uniform transmission line simply amounts to a delay, with no more effects. An extensive parametric analysis supporting this line of reasoning was presented in [31].

\section{B. Experimental results}

Experimental tests were also conducted, considering the complex NUT structure depicted in Fig. 8. Standard $50 \Omega$ coaxial cables were used as the transmission lines making up the NUT: the resulting system is shown in Fig. 9. The ends of the cables were used as testing ports for the sake of simplicity; in practice, testing ports can be obtained by means of any coupling method (capacitive, inductive, etc.) at any position along a cable.

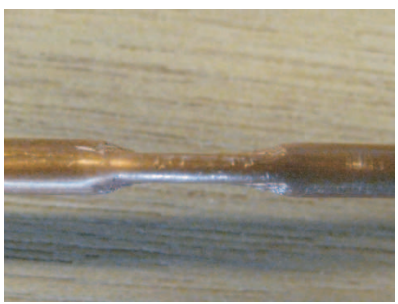

(a)

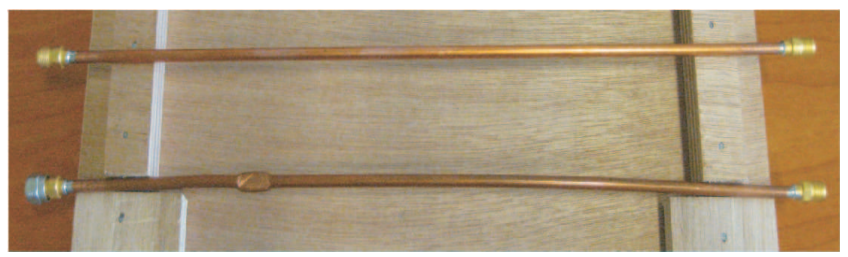

(c)

Fig. 10: The soft fault considered in the experimental validations, as obtained by crushing a $1-\mathrm{cm}$ long portion of a semi-rigid cable. The three pictures present: (a) side and (b) front views of the fault, with the cable reduced to a $2 \mathrm{~mm}$ thickness; (c) the two samples used for the measurements, i.e., the reference semi-rigid cable without fault and the one presenting the fault. They share the same dimensions and features.

Three ends of the NUT were connected to a vector network analyzer, in order to measure its scattering matrix over the frequency range from DC to $800 \mathrm{MHz}$. In order to ensure repeatable results for the NUT with and without fault, two versions of a short section of semi-rigid cable (30 cm long) were manufactured, shown in Fig. 10; one of the two sections was crushed along $5 \mathrm{~mm}$, in order to serve as a soft fault, while the other unaltered section was taken as a reference for the baseline measurement. This kind of approach, where a portion of the faultless NUT is substituted with a faulty portion, rather than directly crushing the original cable, was intended as a way of ensuring reproducible results, in a controllable fashion. As a matter of fact, the direct generation of a fault is hardly reproducible and does not allow to double check results, being an irreversible procedure. Conversely, the risk associated with our approach is to introduce errors due to a not perfectly identical positions of the faultless and faulty samples; we have taken due care in keeping these errors negligible during our tests.

The use of a crushed line is a realistic choice that can emulate any local modification in the characteristic impedance of a transmission line, e.g., a changing distance between the conductors in two-wire cables, partially removed coating, etc. The time-domain response of this kind of fault is shown in Fig. 11 and is fundamentally proportional to the timederivative of the incident signals.

These two $30-\mathrm{cm}$ lines were connected in two steps to the NUT at the location of the red section in Fig. 8; the 


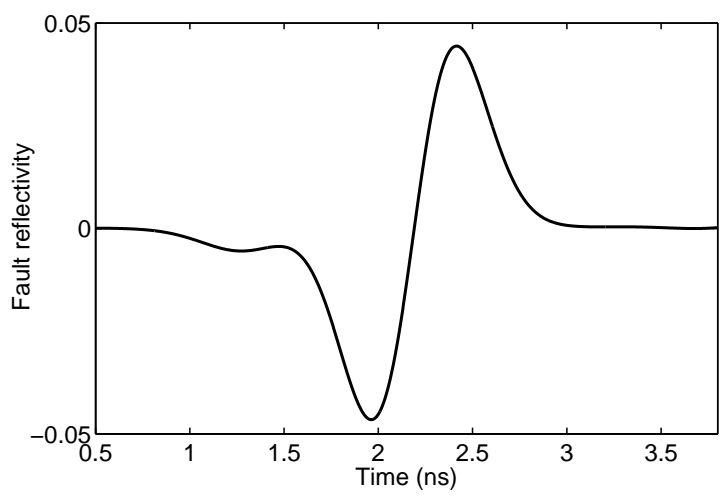

Fig. 11: The time-domain response of the crushed-line fault shown in Fig. 10, as excited by a base-band Gaussian signal with a $-3 \mathrm{~dB}$ bandwidth equal to $800 \mathrm{MHz}$ and peak amplitude equal to one. The derivative nature of the echo is evident from this result.

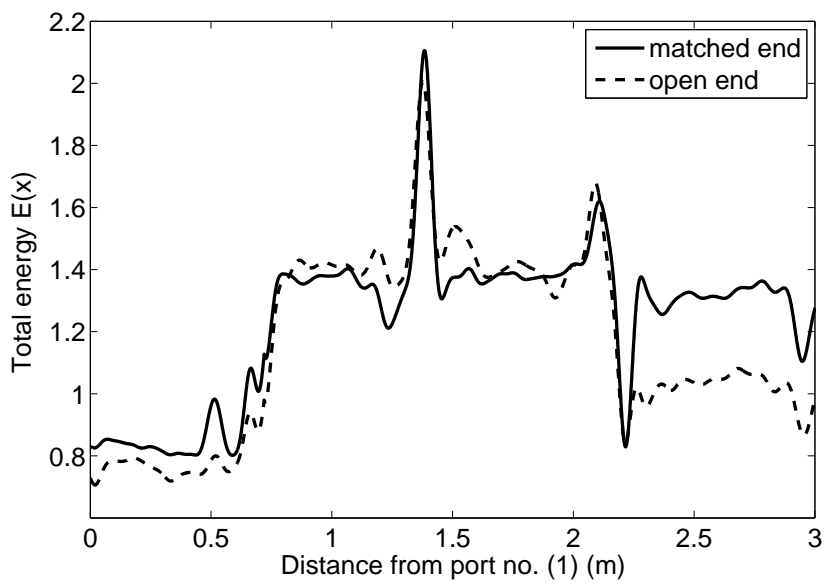

Fig. 12: The total energy diagram corresponding to the NUT in Fig. 9, computed for focusing signals propagating along the path (1)-(3). Open-circuited and impedance-matched terminations are considered for the port number (4).

scattering matrices for the faultless and faulty lines were then measured. The remaining port (4) was either open-circuited or impedance-matched.

Applying the procedure summarized at the end of section III led to the definition of three excitation signals that were subsequently applied to a numerical model of the NUT. From these numerical results we computed the total energy $E(x)$ along the branches in the NUT. This procedure was repeated for the case of port (4) open-circuited or impedance matched. The results are shown in Fig. 12 for the path linking ports (1) and (3).

These experimental results imply that the conclusions drawn from the previous numerical results for a resistive fault are not affected by the fact that soft faults produce echoes that are proportional to the first time-derivative of the impinging signal, i.e., they are frequency dispersive. A formal analysis in [13] proved that in fact frequency-dispersive faults improve the performance of time-reversal-based methods. Furthermore, the results in Fig. 12 also show that the method is hardly affected by the nature of the loads of the NUT; the reason for this phenomenon is that DORT-based methods are fundamentally generating synchronized signals directly converging onto the fault location, without having to rely on the boundary conditions enforced by the NUT. Since most of the focusing energy follows the shortest propagation path between the test ports and the fault position, the nature of the NUT terminations can be expected to have a minor impact. Nevertheless, as demonstrated in [13], NUTs featuring highly reflective terminations can potentially improve the performance of this kind of methods.

\section{CONCLUSIONS}

This paper has proven that fault-location techniques for wire networks can benefit from the use of the DORT method. The physical reasons why this kind of techniques cannot be directly transposed to wire networks have been detailed, proposing a differential version that allows to remove these difficulties. By the same token it appears that this differential DORT technique is intrinsically adapted to the location of soft faults.

Future work will be needed in order to assess the sensitivity of the proposed technique to the fault reflectivity, as well as its robustness with respect to noisy signals propagating along livetested NUTs. Of particular importance will be assessing the impact on fault location of short-term variations in the NUT topology, e.g., due to mechanical vibrations, as well as the analysis of the ability of DORT-based methods in simplifying the problem of multiple faults into a collection of single-fault problems.

\section{REFERENCES}

[1] C. Furse and R. Haupt, "Down to the wire," IEEE Spectrum, vol. 38, no. 2, pp. 34-39, 2001.

[2] T.-W. Pan, C.-W. Hsue, and J.-F. Huang, "Time-domain reflectometry using arbitrary incident waveforms," Microwave Theory and Techniques, IEEE Transactions on, vol. 50, no. 11, pp. 2558 - 2563, November 2002.

[3] P. Tsai, C. Lo, Y. C. Chung, and C. Furse, "Mixed-signal reflectometer for location of faults on aging wiring," Sensors Journal, IEEE, vol. 5, no. 6, pp. 1479-1482, December 2005.

[4] C. Furse, Y. C. Chung, R. Dangol, M. N. G. Mabey, and R. Woodward, "Frequency-domain reflectometery for on-board testing of aging aircraft wiring," IEEE Transactions on Electromagnetic Compatibility, vol. 45, no. 2, pp. 306-315, May 2003.

[5] P. Smith, C. Furse, and J. Gunther, "Analysis of spread spectrum time domain reflectometry for wire fault location," IEEE Sensors Journal, vol. 5, no. 6, pp. 1469-1478, December 2005.

[6] C. Lo and C. Furse, "Noise-domain reflectometry for locating wiring faults," IEEE Transactions on Electromagnetic Compatibility, vol. 47, no. 1, pp. 97-104, February 2005.

[7] S. Naik, C. Furse, and B. Farhang-Boroujeny, "Multicarrier reflectometry," Sensors Journal, IEEE, vol. 6, no. 3, pp. 812 -818, June 2006.

[8] V. Telasula, C. Furse, and C. Lo, "Selection criteria of test signals for correlation-based wire fault analysis," Air Force Research Laboratory, Tech. Rep., 2006.

[9] C. Furse, Y. C. Chung, C. Lo, and P. Pendayala, "A critical comparison of reflectometry methods for location of wiring faults," Smart Structures and Systems, vol. 2, no. 1, pp. 25-46, 2006.

[10] L. Griffiths, R. Parakh, C. Furse, and B. Baker, "The invisible fray: A critical analysis of the use of reflectometry for fray location," Sensors Journal, IEEE, vol. 6, no. 3, pp. 697-706, June 2006.

[11] C.-W. Hsue and T.-W. Pan, "Reconstruction of nonuniform transmission lines from time-domain reflectometry," Microwave Theory and Techniques, IEEE Transactions on, vol. 45, no. 1, pp. 32 -38, January 1997. 
[12] Y.-J. Shin, E. Powers, T.-S. Choe, C.-Y. Hong, E.-S. Song, J.-G. Yook, and J. B. Park, "Application of time-frequency domain reflectometry for detection and localization of a fault on a coaxial cable," Instrumentation and Measurement, IEEE Transactions on, vol. 54, no. 6, pp. 2493 2500, December 2005.

[13] L. Abboud, A. Cozza, and L. Pichon, "A matched-pulse approach for soft-fault detection in complex wire networks," Instrumentation and Measurement, IEEE Transactions on, vol. 61, no. 6, pp. 1719 -1732, june 2012.

[14] C. Buccella, M. Feliziani, and G. Manzi, "Detection and localization of defects in shielded cables by time-domain measurements with UWB pulse injection and clean algorithm postprocessing," Electromagnetic Compatibility, IEEE Transactions on, vol. 46, no. 4, pp. 597 - 605, November 2004.

[15] E. Song, Y.-J. Shin, P. Stone, J. Wang, T.-S. Choe, J.-G. Yook, and J. B. Park, "Detection and location of multiple wiring faults via timefrequency-domain reflectometry," Electromagnetic Compatibility, IEEE Transactions on, vol. 51, no. 1, pp. 131 -138, feb. 2009.

[16] A. Lelong, L. Sommervogel, N. Ravot, and M. Olivas, "Distributed reflectometry method for wire fault location using selective average," Sensors Journal, IEEE, vol. 10, no. 2, pp. 300-310, 2010.

[17] M. Smail, L. Pichon, M. Olivas, F. Auzanneau, and M. Lambert, "Detection of defects in wiring networks using time domain reflectometry," Magnetics, IEEE Transactions on, vol. 46, no. 8, pp. 2998-3001, 2010.

[18] P. Kosmas and C. Rappaport, "A matched-filter fdtd-based time reversal approach for microwave breast cancer detection," Antennas and Propagation, IEEE Transactions on, vol. 54, no. 4, pp. 1257-1264, 2006.

[19] Y. Jiang, J. Zhu, and D. Stancil, "Synthetic aperture radar ghost image cancellation using broadband time reversal averaging techniques," in Microwave Symposium, 2007. IEEE/MTT-S International. IEEE, 2007, pp. 1479-1482.

[20] Y. Jiang, J. Zhu, D. Stancil, and M. Chabalko, "Polarization sensitive time reversal sar imaging in an environment filled with trees," in $A n$ tennas and Propagation Society International Symposium, 2007 IEEE. IEEE, 2007, pp. 4000-4003.

[21] H. Tortel, G. Micolau, and M. Saillard, "Decomposition of the time reversal operator for electromagnetic scattering," Journal of Electromagnetic Waves and Applications, vol. 13, no. 5, pp. 687-719, 1999.

[22] G. Micolau, M. Saillard, and P. Borderies, "DORT method as applied to ultrawideband signals for detection of buried objects," Geoscience and Remote Sensing, IEEE Transactions on, vol. 41, no. 8, pp. 1813-1820, 2003.

[23] C. Prada and M. Fink, "Eigenmodes of the time reversal operator: A solution to selective focusing in multiple-target media," Wave motion, vol. 20, no. 2, pp. 151-163, 1994.

[24] A. Tarantola, Inverse problem theory and methods for model parameter estimation. Society for Industrial Mathematics, 2005.

[25] H. Lev-Ari and A. Devancy, "The time-reversal technique re-interpreted: Subspace-based signal processing for multi-static target location," in Sensor Array and Multichannel Signal Processing Workshop. 2000. Proceedings of the 2000 IEEE. IEEE, 2000, pp. 509-513.

[26] M. Yavuz and F. Teixeira, "Full time-domain DORT for ultrawideband electromagnetic fields in dispersive, random inhomogeneous media," Antennas and Propagation, IEEE Transactions on, vol. 54, no. 8, pp. 2305-2315, 2006.

[27] R. Collin, Foundations for microwave engineering. John Wiley \& Sons, 2007.

[28] E. Iakovleva, S. Gdoura, D. Lesselier, and G. Perrusson, "Multistatic response matrix of a $3-d$ inclusion in half space and music imaging," Antennas and Propagation, IEEE Transactions on, vol. 55, no. 9, pp. 2598-2609, 2007.

[29] M. Born and E. Wolf, Principles of Optics: Electromagnetic Theory of Propagation, Interference and Diffraction of Light. Cambridge University Press, 1999.

[30] C. R. Paul, Analysis of Multiconductor Transmission Lines, K. Chang, Ed. Wiley-Interscience, 1994.

[31] L. Abboud, "Applications of time-reversal techniques to fault-detection in wire networks," Ph.D. dissertation, Supélec, March 2012, available from the authors. 